\title{
CREANDO UN LABORATORIO VIRTUAL PARA DOS ASIGNATURAS BÁSICAS EN GRADOS DE ENFERMERÍA Y PODOLOGÍA
}

\author{
Coronado Carvajal, Carmen ${ }^{1}$ \\ ${ }^{1}$ Universidade da Coruña, \\ Facultad de Enfermería y Podología. \\ ORCID: 0000-0002-4824-6902
}

\section{RESUMEN}

En el curso académico 2020-2021, en la Facultad de Enfermería y Podología se realizaron las clases de docencia expositiva mediante docencia telemática, y algunas clases de docencia interactiva y práctica, principalmente en materias básicas. En estas circunstancias, el e-learning se nos presenta como una estrategia formativa adecuada para resolver el problema educativo con que nos encontramos al inicio del curso. En esta experiencia se describe la transformación a metodología virtual de las clases en grupo pequeño de diez estudiantes en dos materias básicas de Grado en Podología y Grado en Enfermería con competencias del tipo saber. Se ha creado un Laboratorio Virtual de Microbiología mediante la utilización de las herramientas institucionales: Teams para proporcionar la plataforma de reunión síncrona con el alumnado, y Campus Virtual Moodle para gestionar el entorno de enseñanza telemática de manera síncrona y asíncrona. El alumnado ha respondido positivamente a la actividad académica con asistencia síncrona prácticamente del 100\%, realizando el trabajo asíncrono adecuadamente, y en la adquisición de competencias. Por ello, el rendimiento académico alcanzado ha sido excelente. Consideramos que la herramienta desarrollada puede ser muy útil de manera complementaria en las clases presenciales para los próximos cursos.

PALABRAS CLAVE: Laboratorio virtual; Docencia telemática; Microbiología 


\section{CITA RECOMENDADA:}

Coronado Carvajal, Carmen (2021): Creando un Laboratorio Virtual para dos asignaturas básicas en Grados de Enfermería y Podología. En García Naya, J.A. (ed.) (2021). Contextos universitarios transformadores: a nova normalidade académica. Leccións aprendidas e retos de futuro. $V$ Xornadas de Innovación Docente. Cufie. Universidade da Coruña. A Coruña (pág. 95-106).

DOl capítulo: https://doi.org/10.17979/spudc.9788497498180.095

DOl libro: https://doi.org/10.17979/spudc. 9788497498180

\section{ABSTRACT}

In the academic year 2020-2021, at the Faculty of Nursing and Podiatry, expository teaching classes and some interactive and practical teaching classes were held through telematic teaching, mainly in basic subjects. In these circumstances, e-learning appears to us as an adequate training strategy to solve the educational problem that we encountered at the beginning of the course. This experience describes the transformation to virtual methodology of the small group classes of ten students in two basic subjects of the Bachelor's Degree in Podiatry and the Bachelor's Degree in Nursing with knowledge-type skills. A Virtual Microbiology Laboratory has been created using institutional tools: Teams to provide the platform for synchronous meeting with students, and Virtual Moodle Campus to manage the telematics teaching environment in a synchronous and asynchronous manner. Students have responded positively to academic activity with almost $100 \%$ synchronous attendance, performing asynchronous work properly, and in acquiring skills. Therefore, the academic performance achieved has been excellent. We consider that the developed tool can be very useful in a complementary way in the face-to-face classes for the next courses.

KEY WORDS: Virtual laboratory; Telematic teaching; Microbiology 


\section{INTRODUCCIÓN}

En julio de 2020, la Universidade da Coruña (2020) publicó en la web institucional un documento denominado "Orientacións para a programación da actividade docente: curso 2020-2021" con indicaciones dirigidas al profesorado y los centros para organizar la docencia de un curso académico marcado por el transcurso de la pandemia de Covid-19. En dicho documento se indicaba que en aquellos centros en los que no fuera posible mantener la distancia interpersonal de 1,5 metros, se recomendaba optar por un sistema híbrido, que permitiese combinar la docencia presencial con la no presencial.

Esas son las circunstancias que afectaron a la Facultad de Enfermería y Podología, situada en el Campus de Esteiro en Ferrol, por lo que en Junta de Facultad se adoptó un modo de docencia híbrido para el curso académico 2020-2021. En orden de asegurar la calidad de la docencia en los títulos de Grado en Enfermería y Podología que se imparten en el centro, se preservó la docencia presencial para las materias específicas y práctico-clínicas con competencias de saber hacer y saber ser.

En el curso académico 2020-2021 en la Facultad de Enfermería y Podología se realizaron las clases de docencia expositiva mediante docencia telemática, y algunas de las clases de docencia interactiva y práctica, principalmente en materias básicas.

En estas circunstancias, el e-learning se nos presenta como una de las estrategias formativas para resolver el problema educativo con que nos encontramos al inicio del curso académico 2020-2021, pero que también puede ser útil para satisfacer la necesidad de perfeccionamiento constante del profesorado, así como para evitar desplazamientos del alumnado, y conseguir ahorro de tiempo (Cabero, 2006). 


\section{DESCRIPCIÓN DE LA EXPERIENCIA}

En esta experiencia se describe la transformación a metodología virtual de las clases en grupo pequeño de 10 estudiantes en dos materias básicas de Grado en Podología y Grado en Enfermería con competencias del tipo saber.

Para la transformación de esta docencia en las asignaturas "Biología" de Grado en Enfermería, y "Microbiología y Parasitología" de Grado en Podología, se ha creado un Laboratorio Virtual de Microbiología mediante la utilización de las herramientas institucionales: Teams para proporcionar la plataforma de reunión síncrona con el alumnado, y Campus Virtual Moodle como LMS (Learning Management System) o sistema dinámico para gestionar el entorno de enseñanza telemática de manera síncrona y asíncrona.

Para ello hemos usado el recurso "Libro", del Campus virtual de la Universidade da Coruña basado en Moodle 3.0 (Conde Vives et al., 2016), con el que se ha creado un Cuadernillo de prácticas que ha servido de Guía de aprendizaje para el alumnado y para estructurar con coherencia el contenido de las clases.

El Laboratorio Virtual de Microbiología se ha desarrollado en cinco sesiones síncronas de una hora dedicadas secuencialmente a los siguientes contenidos de la materia (Figura 1):

1.- Fundamentos y manejo del microscopio

2.- Observación de tinciones básicas

3.- Preparación de medios de cultivo

4.- Experiencia cualitativa sobre la importancia del lavado higiénico de manos. Siembra de microbiota normal de la piel antes y después de lavado de manos.

5.- Observación de resultados

El contenido metodológico docente de cada una de las sesiones puede verse en la Figura 1. 


\section{Laboratorio Virtual de Microbiología}

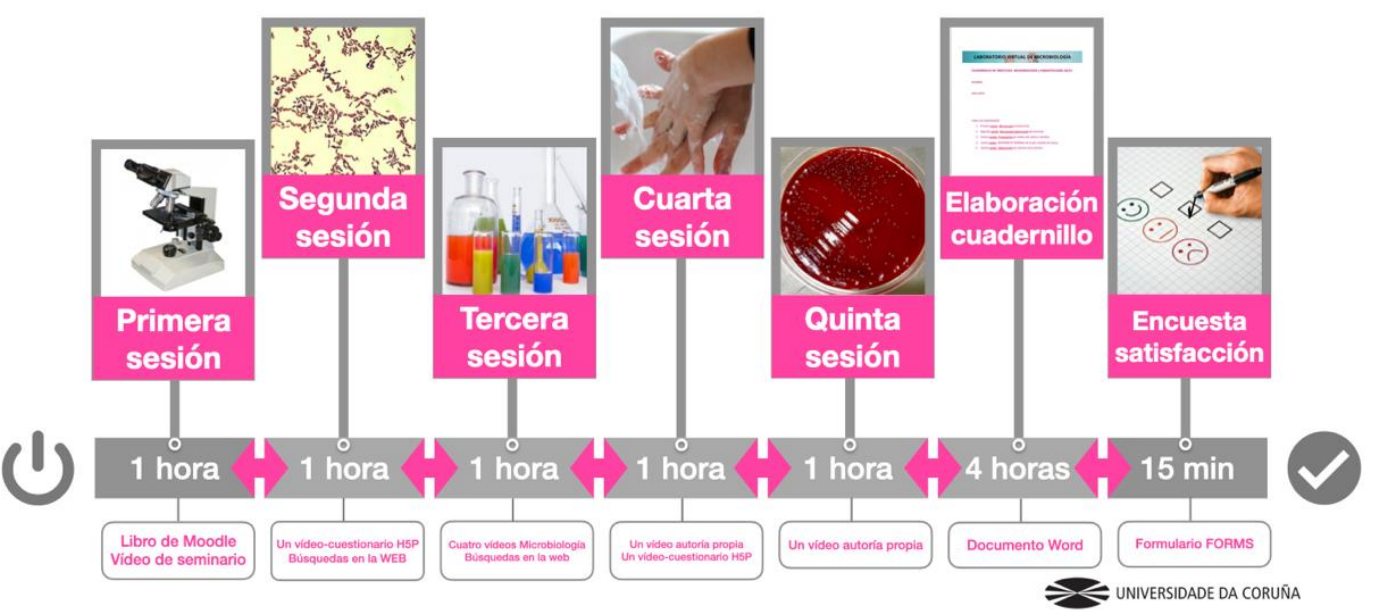

Figura 1. Desarrollo y contenido docente de las sesiones del laboratorio virtual

En las sesiones síncronas, que se realizaron en cinco semanas consecutivas, se mantuvo de manera obligatoria la señal de vídeo y audio de todos los participantes para favorecer la interacción con la profesora y entre pares.

Posteriormente el alumnado tuvo un plazo de dos semanas para realizar de forma individual un informe de laboratorio mediante la utilización del propio libro de Moodle transformado en un archivo Word denominado Cuadernillo de prácticas disponible en el Campus virtual de las materias, y que debería entregarse a la profesora mediante una "Tarea" del campus virtual. El tiempo estimado de trabajo del estudiante de forma asíncrona fue de 4 horas.

Al final de la última sesión, se solicitó al alumnado que cubriese de forma anónima un formulario de Forms sobre su experiencia en la e-actividad. Dicho formulario estuvo disponible una semana y el tiempo estimado para responderlo fue de 15 minutos. Para preservar el anonimato de los participantes, se codificaron las encuestas con las siglas EP para estudiantes de Podología y EE para estudiantes de Enfermería, seguidas de un número de orden. 
La evaluación de esta actividad se explicó adecuadamente al alumnado en las reuniones síncronas. De forma que la asistencia a las cinco sesiones aportaría el $30 \%$ de la calificación de la e-actividad, el resultado de cada uno de los dos vídeo-cuestionarios incluidos en las sesiones aportaría el 10\%, independientemente de que se realizase de manera síncrona 0 asíncrona, y finalmente el informe escrito sobre el Cuadernillo de prácticas contribuiría con el $50 \%$ del total de la calificación.

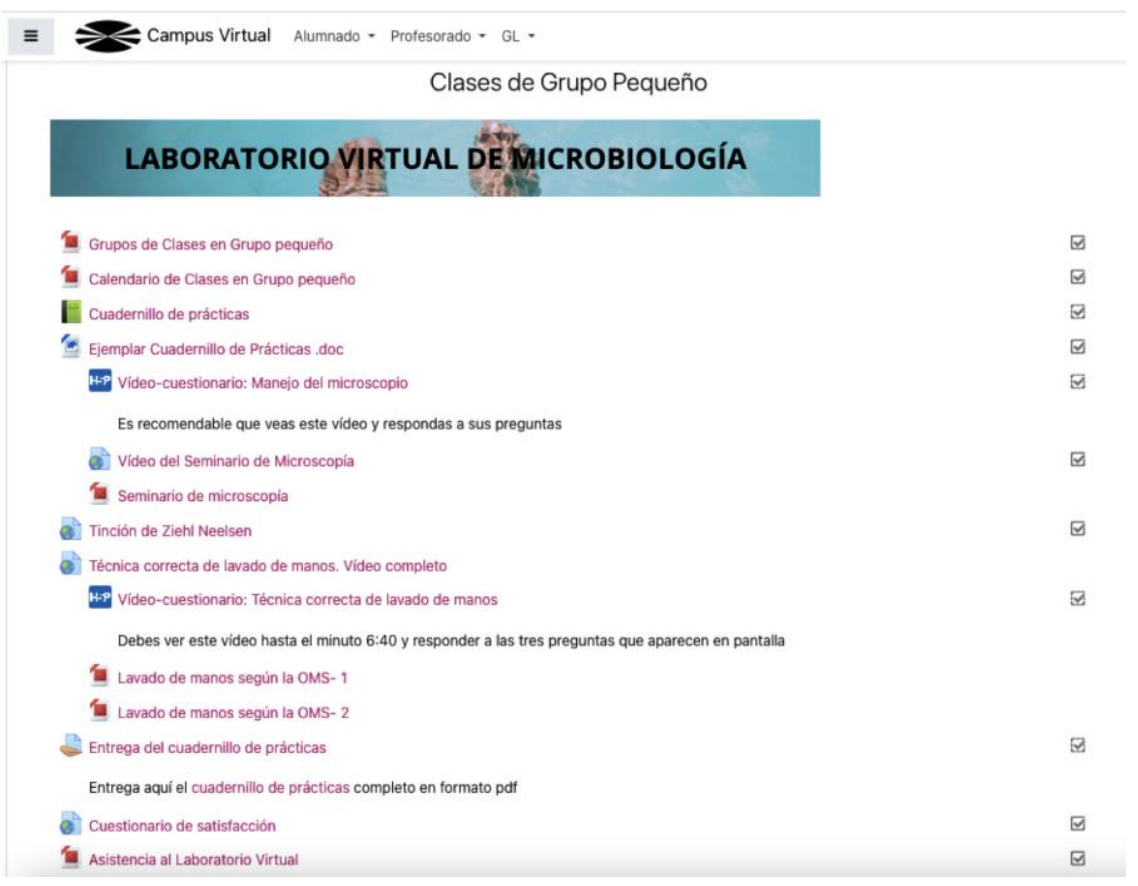

Figura 2. Vista de la actividad en el Campus virtual UDC

En la Figura 2 se muestra una vista de la sección correspondiente al Laboratorio Virtual de Microbiología en el Campus virtual UDC. Se han utilizado recursos metodológicos de H5P (Proyecto impulsado por la comunidad; https://h5p.org) integrados en la última versión del Campus virtual UDC, y recursos de contenido obtenidos de la OMS sobre el lavado de manos, y de diversos docentes de Microbiología en la Universidad de Sevilla, Universidad de Salamanca y Universidad Miguel Hernández de Elche. 


\section{RESULTADOS}

En esta experiencia docente participaron 53 alumnos/as de Grado en Podología y 61 alumnos/as de Grado en Enfermería. La actividad académica se ha seguido prácticamente con el $100 \%$ de asistencia por ambos cursos, y el rendimiento académico alcanzado ha sido excelente. En la Figura 3 se presentan los resultados académicos del grupo de Podología donde vemos que la media del grupo se sitúa en 9,47 puntos sobre 10. Esta calificación aportó el $20 \%$ de la nota final de la asignatura.

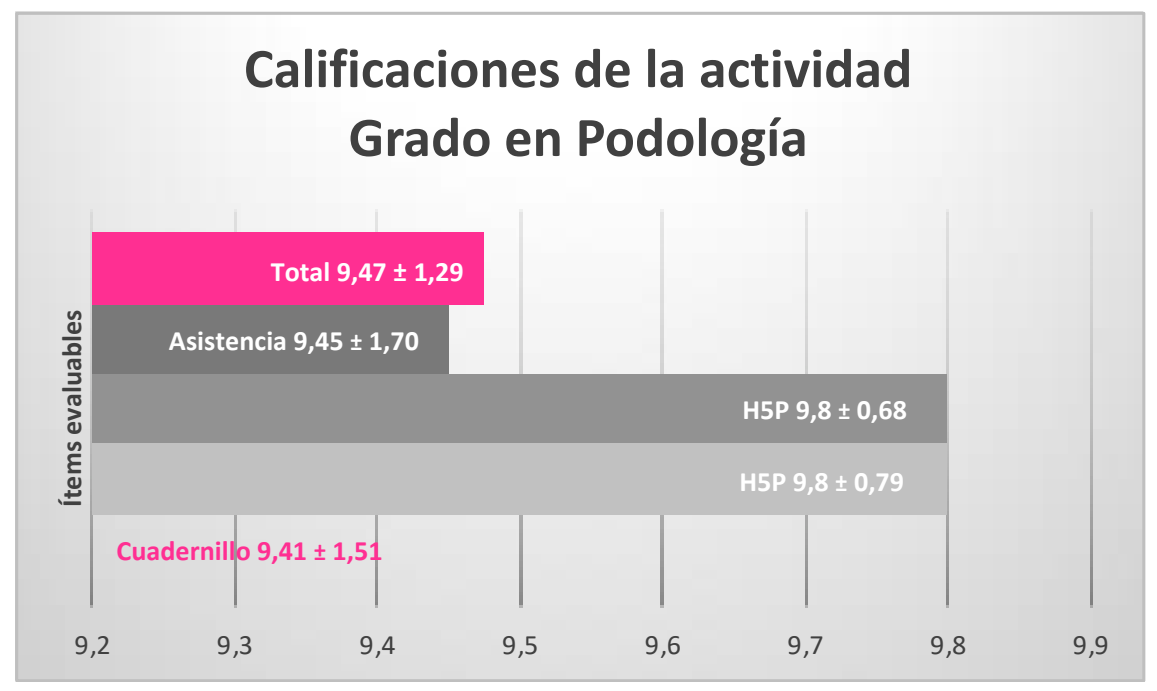

Figura 3. Calificaciones obtenidas en la actividad académica por el alumnado de Grado en Podología

El 55,7\% del estudiantado de Enfermería y el 73,6\% del estudiantado de Podología respondió la encuesta de satisfacción anónima. Esta encuesta se estructuró en cuatro bloques en los que se preguntaba por:

1.- La planificación de la actividad

2.- El desarrollo de la misma

3.- Los resultados, y

4.- La coordinación y tutoría realizada por la docente.

En la Figura 4 se presentan los resultados del grupo de Podología donde: 
El 100\% del estudiantado estuvo de acuerdo (DA) o totalmente de acuerdo (TDA) en que la actividad se planificó con tiempo suficiente y con toda la información precisa, y en que los objetivos de aprendizaje estaban definidos con claridad y se ajustaban a los objetivos y contenidos de la asignatura.

El 97,4\% del alumnado admitió que el Laboratorio virtual les había permitido conocer el trabajo del laboratorio de Microbiología, la importancia para los sanitarios del lavado de manos, y la presencia de microorganismos en el cuerpo humano.

Con respecto a las labores de tutorización, ese mismo porcentaje opinó que se les habían explicado con claridad los objetivos de la e-actividad, que la docente se había involucrado en el desarrollo de las clases y que tuvieron su apoyo en todo momento.
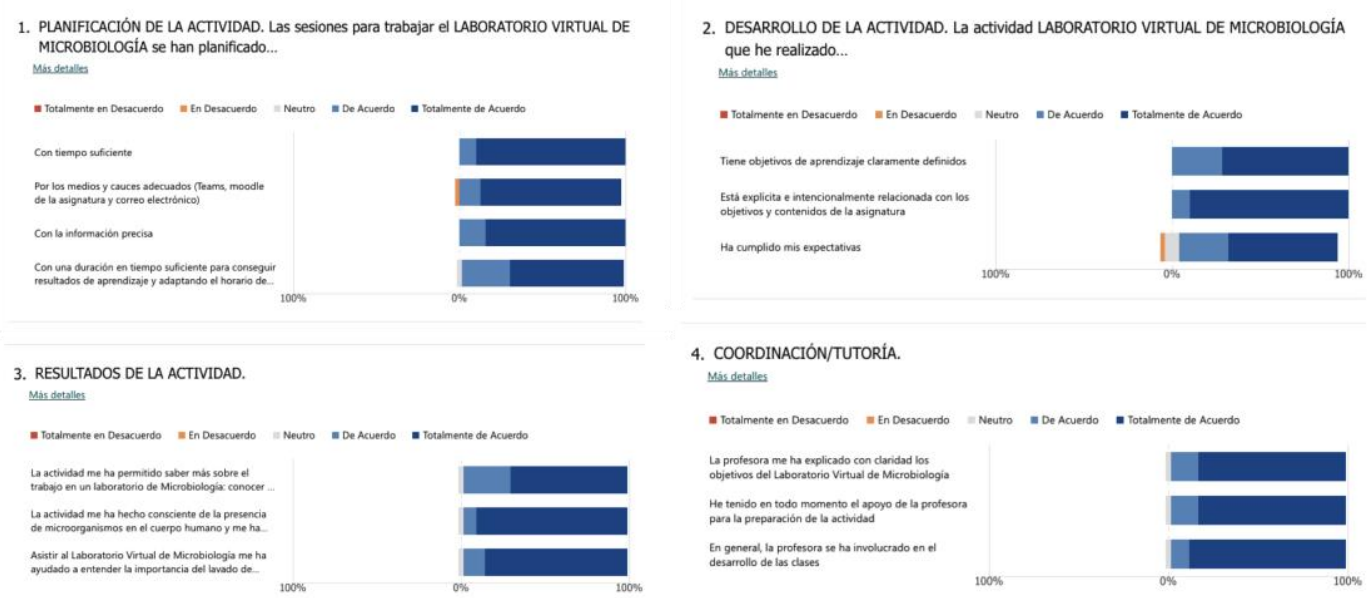

Figura 4. Resultados de la encuesta de satisfacción del alumnado. Grado en Podología.

A partir del análisis cualitativo de las aportaciones recibidas en la pregunta abierta del final del cuestionario de satisfacción, se pueden destacar cuatro temas emergentes que se describen a continuación.

La presencialidad es importante para la enseñanza práctica en nuestro alumnado, como podemos leer en las aportaciones de los siguientes participantes en la experiencia: 
Nada que mejorar (...). La única pega que puedo poner es que no se pudiesen realizar de manera presencial estas prácticas, ya que no es lo mismo por Teams. EP3

Esta bien planteado pero creo que es necesario hacerlo presencialmente ... EP14

La actividad ha cumplido el objetivo de lograr un nivel adecuado de interactividad, como nos indican los estudiantes:

La profesora (...), nos ha propuesto diversas actividades con el propósito de interaccionar con los alumnos y se han desarrollado con el tiempo suficiente para entenderlo todo y aclarar dudas. EP1

El laboratorio virtual además de ayudar a afianzar conocimientos, ha sido muy entretenido y dinámico. EP9

Su (de la profesora) método de hacer diferentes tipos de tareas ayuda a aprender mejor. EP10

El estudiantado valora el empleo de nuevas tecnologías que resulten atractivas como los vídeo-cuestionarios:

Los vídeo-cuestionarios me parecieron muy útiles porque puedes comprobar lo que has aprendido. EE1

(...) dentro de la situación que nos ha tocado vivir, las prácticas a través de la plataforma me parece que han estado muy bien organizadas y se han hecho muy amenas (...) EP6 
Nuestros estudiantes saben valorar el esfuerzo docente que realizan el profesorado:

No hay nada que decir, se nota que hay esfuerzo y trabajo por parte de la profesora. Para mí fue un 10 en todo, valió la pena. EP7

Me ha gustado mucho profe, muchas gracias por ponerle tanta dedicación y paciencia. EP13

\section{CONCLUSIONES}

A continuación, se exponen las principales conclusiones de este trabajo de investigación docente:

- Se ha conseguido transformar eficientemente una actividad docente presencial en actividad telemática asistida con las herramientas institucionales para este formato docente.

- Los resultados académicos alcanzados por los estudiantes han sido muy satisfactorios en ambos grupos de clase/título.

- El alumnado ha respondido positivamente con asistencia síncrona muy elevada, realizando el trabajo asíncrono adecuadamente, y en la adquisición de competencias.

- Mantenemos que la presencialidad en el laboratorio de Microbiología es esencial, si las circunstancias sanitarias lo permiten.

- Como propuesta de mejora, sería deseable disponer de una aplicación interactiva para laboratorio virtual de Microbiología, que nos permitiera emular la realización de tareas propias de la disciplina.

- Finalmente, consideramos que la herramienta desarrollada puede ser muy útil de manera complementaria en las clases presenciales para los próximos cursos. 


\section{REFERENCIAS}

Cabero Almenara, J.(2006). Bases pedagógicas del e-learning. Revista de Universidad y Sociedad del Conocimiento UOC, 3 (1), pp. 1-10. Disponible en: www.uoc.edu/rusc

Conde Vives, J. V., García Luna, D., García Rodríguez, J., Hermiz Ramírez, A., Moreno López, J. J., Muñoz Solís, P. L., Osorio Navarro, A. Manual Moodle 3.0 para el profesor. Universidad Politécnica de Madrid. 2016. Disponible en: http://0a.upm.es/42658/1/manual_moodle_30.pdf

Organización Mundial de la Salud. Hand higiene Technical Referente Manual. 2009. Disponible en: https://apps.who.int/iris/bitstream/handle/10665/102537/WHO_IER_PSP_2009.02_spa.pdf ;jsessionid=6A02311111D5D072BF1ED53979D6A7EB? sequence $=1$

Recurso H5P. https://docs.moodle.org/all/es/H5P ; https://h5p.org/

Recurso online Student Consult. Microbiología médica de Murray. 2021. Disponible en: https://studentconsult.inkling.com/

Universidad Autónoma de Nuevo León. México. Microscopio. Disponible en: https://www.youtube.com/watch?v=70gEkF0kj1c

Universidade da Coruña. Orientacións para a programación da actividade docente: curso 20202021. 2020. Disponible en: https://www.udc.es/export/sites/udc/covid19/_galeria_down/orientacions.pdf_2063069294.pdf

Universidad de Sevilla. Secretariado de recursos audiovisuales y nuevas tecnologías. Dpto. Biología celular y Dpto. Fisiología. Conocimiento, manejo y aplicaciones del microscópico óptico de campo claro en las prácticas de laboratorio de biología. Disponible en: https://www.youtube.com/watch?v=905Nbn1VYK4

Universidad de Sevilla. Secretariado de recursos audiovisuales y nuevas tecnologías. Dpto. Microbiología. Técnicas básicas en el laboratorio de Microbiología. Preparación de medios de cultivo. Disponible en: https://www.youtube.com/watch?v=miga09gVMyY 
Universidad de Sevilla. Secretariado de recursos audiovisuales y nuevas tecnologías. Dpto. Microbiología. Técnicas básicas de Microbiología. Siembra y aislamiento de bacterias. Disponible en: https://www.youtube.com/watch?v=-TnHCd4sY24

Universidad Miguel Hernández de Elche. Dpto. Producción vegetal y Microbiología. Preparación de medios de cultivo. Disponible en: https://www.youtube.com/watch?v=YGoPu0cn9ms Universidad de Salamanca. Dpto. Microbiología y Genética. Técnicas básicas de Microbiología: Aislamiento y siembra en estría de una bacteria. Disponible en: https://www.youtube.com/watch?v=6NckVGCobwY 